\title{
BMJ Open Family-building decision aid and planning tool for young adult women after cancer treatment: protocol for preliminary testing of a web-based decision support intervention in a single-arm pilot study
}

Catherine Benedict (D , , ${ }^{1,2}$ Jennifer S Ford, ${ }^{3}$ Lidia Schapira, ${ }^{1,2}$ Pamela Simon, ${ }^{4}$ David Spiegel, ${ }^{1,2}$ Michael Diefenbach $^{5}$

To cite: Benedict C, Ford JS Schapira L, et al. Familybuilding decision aid and planning tool for young adult women after cancer treatment: protocol for preliminary testing of a web-based decision support intervention in a singlearm pilot study. BMJ Open 2019;9:e033630. doi:10.1136/ bmjopen-2019-033630

- Prepublication history and additional material for this paper are available online. To view these files, please visit the journal online (http://dx.doi. org/10.1136/bmjopen-2019033630).

Received 13 August 2019 Revised 12 November 2019 Accepted 04 December 2019

Check for updates

(C) Author(s) (or their employer(s)) 2019. Re-use permitted under CC BY-NC. No commercial re-use. See rights and permissions. Published by BMJ.

For numbered affiliations see end of article.

Correspondence to Dr Catherine Benedict; cbenedict@stanford.edu

\section{ABSTRACT}

Introduction Many young adult female (YA-F) cancer survivors who received gonadotoxic therapy will experience fertility problems. After cancer, having a child will often require assisted reproductive technology (ART), surrogacy or adoption. However, there are significant informational, psychosocial, financial and logistical barriers to pursuing these options. Survivors report high rates of decision uncertainty and distress related to family-building decisions. The aim of this study is to pilot test a webbased decision aid and planning tool for family-building after cancer.

Methods and analysis The pilot study will use a singlearm trial design to test the feasibility and acceptability (aim 1) and obtain effect size estimates of the decision support intervention (aim 2). The target sample size is 100 . Participants will include YA-F survivors (aged 18-45 years) who are post-treatment and have not completed desired family-building. A longitudinal prepost design will be conducted. Participants will complete three psychosocial assessment surveys over a 3-month time period to track decisional conflict (primary outcome) and cognitive, emotional, and behavioural functioning (secondary outcomes). After completing the baseline survey ( $\mathrm{T} 1$; preintervention), participants will have access to the decision aid website. Postintervention surveys will be administered at 1-month (T2) and 3-month (T3) follow-up time points. Feasibility and acceptability metrics will be analysed. Pairwise $t$-tests will test mean scores of outcome variables from T1 to T2. Effect size estimates (Cohen's d) will be calculated. Google analytics will evaluate user engagement with the website over the study period. Baseline and follow-up data will examine measures of feasibility, acceptability and intervention effect size.

Ethics and dissemination This will be the first test of a supportive intervention to guide YA-F cancer survivors in family-building decisions and early planning. Study findings will inform intervention development. Future directions will include a randomised controlled trial to test intervention efficacy over a longer time period.

Trial registration number NCT04059237; Pre-results.
Strengths and limitations of this study

- This will be the first test of a supportive intervention to guide young adult female cancer survivors in family-building decisions and early planning, thus helping them to achieve parenthood goals after cancer.

- The study will lead to the first longitudinal assessment of decisional conflict about family-building after cancer.

- As this is a pilot study, outcome variables will be measured over a 3-month time period and we will not be able to detect if decisions need to be revisited after failed family-building attempts or if decision support needs change over a longer period of time.

- This study does not address system, provider and partner/family-level factors that may affect the decision-making of young adult female cancer survivors related to family-building or impacting their decision support needs.

- A control group is not included in this pilot trial; however, findings will inform a randomised controlled trial to test intervention efficacy over a longer time period.

\section{INTRODUCTION}

Many young adult female (YA-F) cancer survivors have received gonadotoxic therapy and are infertile, at-risk for premature ovarian failure or unable to carry a pregnancy. ${ }^{1}$ As a result, family-building after cancer often requires assisted reproductive technology (ART), surrogacy or adoption. Most YA-F survivors hope to have children someday and risks related to reproductive potential is a distressing survivorship issue. ${ }^{2}$ Pretreatment counselling on infertility risks and fertility preservation (such as egg or embryo freezing) is an established part of care for reproductive aged patients, ${ }^{34}$ 
but follow-up counselling after treatment is completed is often missing or inadequate. ${ }^{5}$ Most survivors are unsure of their reproductive potential post-treatment and distressed about their chances for achieving parenthood. ${ }^{6-8}$ Failure to attend to these issues places young women at risk for experiencing unexpected early menopause, thereby eliminating chances for having a biologically related child, and/ or being unprepared for the challenges of ART, surrogacy or adoption, which include medical, emotional, legal and financial complications.

\section{Decision support needs}

For YA-Fs that experience treatment-related fertility problems, family-building options typically include in vitro fertilisation (IVF) or surrogacy with fresh, frozen or donated gametes, or adoption (domestic or international). Decision-making about these options is complex. Even when survivors are informed about their options, they still face the difficult task of making decisions within a highly uncertain situation. This involves weighing the pros and cons of different options based on inexact estimates of fertility potential and timeline, likelihood of ART success or potential health risks, complex legal procedures for surrogacy and adoption, and unknown bureaucratic difficulties. Cost estimates vary greatly depending on the number of IVF cycles needed to achieve pregnancy for the survivor or surrogate, whether donated gametes will be used, or agency/lawyer fees with surrogacy and adoption. Decisions are preference-sensitive and women must decide what factors matter most to them (and their partners), while weighing potential risks and benefits.

Although it is well accepted that fertility-related distress after cancer is common, particularly among females, ${ }^{9}$ there is a lack of support resources for post-treatment survivors. Multiple cancer and fertility decision aids and support interventions exist for YA-Fs considering pretreatment fertility preservation. ${ }^{10-15}$ Consistent with the decision science literature ${ }^{16}$ these studies demonstrate the benefit of decision aids for YA-Fs facing fertility decisions. However, none of them include comprehensive information about decisions that must be made after cancer treatment is completed. Fertility preservation decisions at the time of a cancer diagnosis is also a discrete decision-making time period with clear decision options (yes/no), whereas family-building decisions are more complex with more options and barriers to consider, such as choosing between IVF or surrogacy (with/without fresh/frozen/donated gametes and laws that vary by state) or domestic or international adoption (with multiple country options). Family-building may be a more iterative decision-making process if 'first-choice' options do not pan out, such as after failed IVF attempts. Thus, more support may be required to plan ahead, set expectations, and to help survivors translate a decision into preparatory action to mitigate or prevent potential challenges. For example, survivors that hope to have a biologically related child should have their fertility tested to learn if they have a shortened reproductive timeline due to cancer treatment effects and to avoid unexpected premature ovarian failure.
Those hoping to pursue surrogacy should learn about regulatory laws in the state they live in, or similarly for adoption regarding domestic/international laws and regulations. It may also be important to plan ahead for financial costs.

\section{Development of a web-based decision aid tool}

Benedict $e t$ al developed an interactive, web-based decision aid and planning tool for family-building after cancer, named 'Roadmap to Parenthood'. To our knowledge, this is the first evidence-based resource to help YA-Fs make decisions about family-building after cancer and guide self-management towards 'next steps' for fertility care and family-building preparation. The tool was designed to help YA-F survivors learn about options for familybuilding post-treatment, identify personal priorities and goals (values clarification), and initiate early action to plan ahead. It was developed based on extensive pilot work $^{7817-23}$ and theory, ${ }^{2425}$ following healthcare decision aid guidelines ${ }^{1626}$ and patient-centred design principles. ${ }^{27}$

\section{Premise of the study and objectives}

After completing cancer treatment, young female survivors report a lack of knowledge coupled with high levels of distress about their fertility, as well as unmet decision support needs related to their pursuit of parenthood. To address this gap, this study protocol will pilot test the first theoretically driven, evidence-based decision aid and planning tool for family-building after cancer. The specific aims are: 1) to examine feasibility and acceptability of a decision aid intervention and 2) obtain effect size estimates of the intervention using a single-arm, pre-post study design. We hypothesise that the intervention will be feasible to conduct, and the web-based tool will be acceptable to participants. We also hypothesise the intervention will help post-treatment YA-F survivors manage decisional conflict (primary outcome), feel informed about fertility and family-building options, manage negative cognitions and emotions, and feel more confident in addressing concerns/ risks (secondary outcomes and process variables).

\section{METHODS}

This study will take place at Stanford University School of Medicine in Palo Alto, California, USA. The Standard Protocol Items: Recommendations for Interventional Trials reporting guidelines were followed including compliance with the WHO Trial Registration Data Set (see online supplementary appendix 1$).{ }^{28}$ The principal investigator (PI) (Benedict) will oversee the training of study research coordinators and conduct weekly meetings to ensure fidelity to study procedures. Protocol amendments will be communicated to relevant parties (eg, co-investigators, participants). Self-reported adverse events that are caused by the trial will be tracked and reported. Stanford University is the study sponsor.

The overall goal of this work is to pilot test a decision aid intervention to assist young women in making decisions about family-building after cancer when ART, surrogacy or 
adoption may be needed, and to prompt them to consider preparatory actions to plan for potential challenges. To achieve the research objectives, we will conduct a single-arm pilot trial to evaluate the feasibility and acceptability of the tool and study procedures (aim 1) and obtain effect size estimates for a future large-scale trial involving the tool (aim 2).

\section{Participants}

The target sample size is 100 participants. Inclusion criteria includes: female, ability to speak and read English, aged 18-45 years, completed cancer treatment with risks of gonadotoxic effects (eg, systemic chemotherapy, pelvic radiation and surgery involving reproductive organs), desire future children or are uncertain of family-building plans, have access to the internet and use of a computer, tablet or smartphone, and reside in the USA. Patients with significant physical or mental disability that prevents completion of study activities will be excluded. Survivors on adjuvant maintenance or endocrine treatment, such as tamoxifen, will not be excluded because clinical guidelines may allow treatment delay or hiatus to accommodate fertility preservation, egg extraction for surrogacy or pregnancy for some patients, and because patients may be interested in surrogacy with frozen or donated eggs/embryos or adoption. Participants will be stratified by age (18-25 years, 'emerging adult'; 26-39 years, 'young adulthood' and 40-45 years, post-young adulthood but within the reproductive age range), ${ }^{29} 30$ as well as partnership status (single vs coupled) and parenthood status (nulliparous vs parous). This study will only include females, which allowed us to develop educational material tailored to female reproductive health and healthcare decision-making, as well as a website design that would be appealing to women as opposed to a more neutral design for both men and women. Females also report greater unmet information needs and more negative emotional reactions with fertility uncertainty, and face more complex and costly procedures with ART, compared with males; and have unique needs related to reproductive health and pregnancy concerns (eg, questions about ovarian reserve, reproductive timeline, and safety of pregnancy). 83132

\section{Study design}

A longitudinal pre-post intervention design will be conducted (figure 1). Survey questionnaires will be administered at baseline (T1; pre-intervention) and at 1-month (T2) and 3-month (T3) follow-up time points. These time points were chosen to allow users to review the content of the decision aid website and have time to access medical records, finances or insurance information, if needed, to better assess decision options, complete interactive features, and optimise engagement with the site.

\section{Description of the intervention}

The intervention consists of a web-based patient decision aid and planning tool for family-building after cancer (Roadmap to Parenthood). The primary purpose of the tool

\section{- Hospital-based \\ Recruitment \\ - Partnership with patient organizations \\ - Social media ads}

- Confirm eligibility

- Conduct informed consent

\section{Enrollment \\ (Target $\mathrm{N}=100$ )}

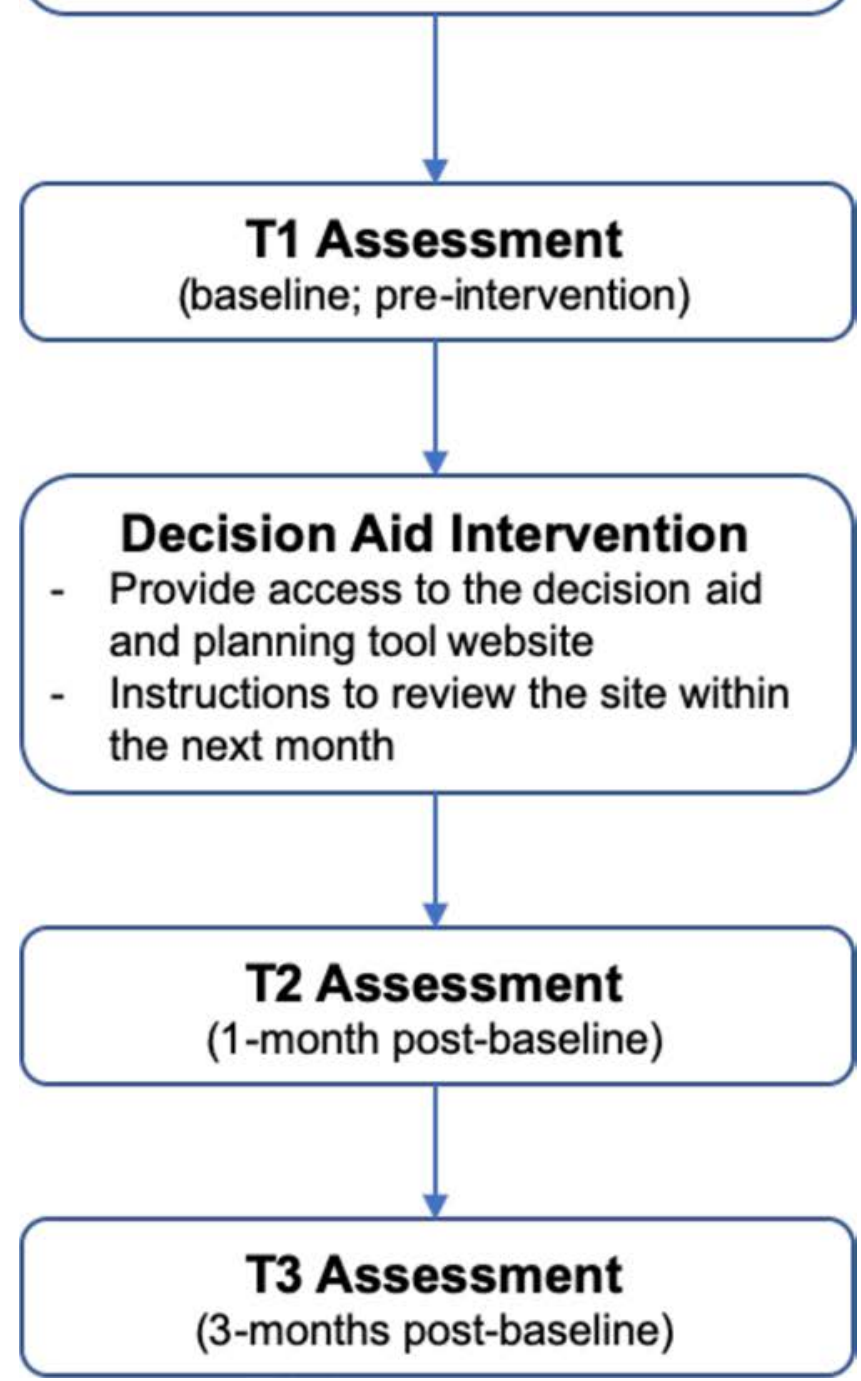

Figure 1 Study design and participant flow. A longitudinal prepost intervention design will be conducted in which participants $(n=100)$ complete three survey questionnaires at baseline (T1; pre-intervention) and at 1-month (T2) and 3-month (T3) follow-up time points (postintervention).

is to support YA-F cancer survivors in making decisions about family-building after treatment and prompt early planning and preparatory action. Family-building decision options include natural conception (if possible, after 
gonadotoxic treatment), IVF with fresh/frozen gametes, IVF with donor gametes, surrogacy with fresh/frozen/ donated gametes, and adoption or fostering (ie, five main decision options); post-treatment fertility preservation was added as an additional option for YA-Fs who wish to have a biologically related child, but are not yet ready to start family-building, to mitigate risks of premature ovarian failure. Although family-building may be years away for some participants, the aim of the intervention is to provide education about options and support early decision-making to prompt actions that may need to be taken immediately or in the short-term to achieve longterm family-building goals. For example, YA-Fs who desire a biologically related child should have their fertility evaluated, understand their expected timeline of reproductive potential and may consider post-treatment fertility preservation in the case of diminished ovarian reserve. While the intervention aims to guide early decision-making and preparatory action aligned with YA-Fs' current desires, as with many long-term plans for managing health risks and goals, family-building preferences may change over time and decisions may be revisited including iterative use of the intervention.

The intervention includes information pages about cancer treatment effects on fertility and alternative family-building options, provides information about recommended 'next steps' to consider (eg, 'questions to ask your oncologist', 'questions to ask a reproductive specialists' and 'financial planning'), interactive features (eg, values-clarification exercise and selfassessment exercise of support needs and resources) and an extensive resource page to link to more in-depth information across a range of topics from trusted sources (eg, professional organisations, finding a fertility clinic that specialises in oncofertility, available grants and financial support resources, and outlets to access peer support and professional counselling). Participants are expected to engage with the website by reviewing educational material and options for actionable next steps, and completing interactive exercises to evaluate decision-making readiness, clarify values and identify unmet decision support needs. They may also review stories depicting the experiences of other YA-F cancer survivors pursuing each family-building option. Content of the intervention and development of the website was based on semi-structured interviews $(n=25)$ exploring YA-F cancer survivors' post-treatment decision support needs and preferences for support related to fertility and family-building (NCI R03CA212924; manuscript under review). The website underwent usability testing to ensure an optimal human-centred design interface (NCI K07CA229186).

The intervention was designed for individual use. Although social support is often an important component of decision-making, there may also be a downside of providing access to peer support through an anonymous online community such as the risk of spreading misinformation and emotional contagion. ${ }^{33} 34$ Several features were created to guide users to find support that meets their needs (ie, 'finding support', 'talking to your partner' and resource links to support groups and relevant patient organisations).

The intervention may serve as a one-time decision support resource or to support iterative decisionmaking based on users' experiences, needs, and outcomes of intermediary steps. For those with greater baseline awareness of their fertility status and knowledge about options, the intervention may serve to confirm preconceived choices and prompt preparatory action with limited engagement with the website. Other survivors may struggle with decisional processes and return to the website to review information, re-engage interactive exercises or revisit options as new information is obtained. For example, many women prioritise having a biologically related child but may learn they are infertile or have a low chance of success with IVF after obtaining a fertility evaluation; and they may re-engage with the website to re-evaluate options and preferences in light of new information. In the longer term, women may restart the decision process if they are unsuccessful with a chosen family-building path, such as after failed IVF attempts, or the challenges become too great. Single women may revisit decision-making when involving a partner. Based on this conceptualisation of decision-making processes, the design of the website included support for iterative engagement such that the information architecture was set up to allow users to have maximum control over the user journey and easily circle back to content about alternative family-building options.

The design and development of the decision aid tool followed guidelines from the International Patient Decision Aid Society ${ }^{26}$ and the Ottawa Decision Support Framework for developing patient decision aids ${ }^{36}$; and were in compliance with the required components of a patient decision aid. ${ }^{38}$ We also followed guidelines for designing websites for low health literacy and for culturally diverse populations. ${ }^{39}$ The intervention is designed to be inclusive of all sexual orientations and for single women. A responsive design website was used because it can adapt layout and content across digital devices, creating the same user experience for all operating systems. ${ }^{40}$

\section{Recruitment and enrollment}

Recruitment will be conducted through the Stanford Comprehensive Cancer Center and Lucile Packard Children's Hospital including the Stanford Adolescent and Young Adult Cancer programme. Patients may also be referred from clinical partners from across the healthcare system and study advertisements. We will obtain a Waiver of Authorisation for Recruitment to conduct chart reviews and telephone screens to determine preliminary eligibility. The primary treating oncologist of patients identified through chart review will be contacted to request permission to invite the 
patient for research participation. After receiving physicians' approval, an initial recruitment letter will be sent and a research coordinator will make follow-up phone calls 1 week later to describe the study in more detail, confirm eligibility and complete enrolment for those eligible and interested in participating.

We will also recruit through social media and collaborations with young adult cancer patient organisations. Using social media and remote recruitment and consenting procedures is advised for recruiting young adult patient populations. ${ }^{41}{ }^{42}$ Institutional review board (IRB)-approved ads will be posted on relevant social media pages (eg, Facebook, Instagram) and distributed through patient organisations' listservs. For ads that we post (and not partnering organisations), we will set security settings that do not allow participants or others from the public to post publicly on the ad or social media page. The ads/posts will instruct viewers to click on a link directing them to a secure, Health Insurance Portability and Accountability Act (HIPPA) compliant website to provide contact information and a research coordinator will follow-up as part of recruitment procedures.

Recruitment and consenting conversations will involve a thorough discussion of study aims and procedures, as well as the opportunity for potential participants to ask questions and confirm understanding. Following recruitment conversations (in-person or telephone), a link to access the informed consent document (see online supplementary appendix 2) online will be emailed to eligible and interested patients or viewed in-person on a tablet. The PI and research coordinators will complete informed consent and enrolment procedures. Consenting will include having a patient read an informed consent document and provide an electronic signature via Research Electronic Data Capture (REDCap), an online HIPPA-compliant platform approved for use with 'high risk' data; hosted by the Stanford Center for Clinical Informatics. Participants will be offered a paper-based copy of the informed consent document, if preferred.

\section{Procedures}

Participants will complete three psychosocial assessment surveys (T1-T3) to track decisional conflict (primary outcome) and cognitive, emotional and behavioural functioning (secondary outcomes), and a priori covariates and process variables including sociodemographic and medical characteristics, uncertainty management preference, self-efficacy, financial well-being and health literacy (table 1). Surveys will be administered remotely via REDCap. Following completion of the baseline survey (T1), participants will be emailed a link to access the decision aid and planning tool website with a prompt to review the site within the next couple of weeks. A research coordinator will contact participants 2 weeks after providing access to the intervention to assess whether participants visited the website (intervention adherence) and/or evaluate reasons for non-use (nonadherence). Follow-up surveys will be emailed 1 and 3 months postbaseline (T2 and T3, respectively). Access to the website will remain open for the duration of the study. The research coordinator will monitor survey completion and follow up with participants with missing surveys to optimise data collection. A small compensation will be given to participants for completion of study activities (US\$10).

\section{Measures}

To complete study aim 1, feasibility and acceptability metrics will be collected. Feasibility of the decision aid intervention and study design will be evaluated with rates of eligibility, enrolment and attrition. Reasons for refusal to participate will be tracked and evaluated. Acceptability will be evaluated using survey completion rates and web analytics to track access to website modules and completion of exercises including time spent and click stream data. Acceptability will also be assessed with survey questions at T2 and T3 to assess self-reported website use, the eHealth Impact Questionnaire (eHIQ) Information and Presentation subscale and open-ended questions about overall impressions, likes/dislikes, and suggestions for improvement.

To complete study aim 2, effect size estimates will be calculated for reduction of decision conflict (primary outcome; Decisional Conflict Scale (DCS) ${ }^{43}$ ). Effect size estimates will also be calculated for secondary outcomes including unmet fertility information needs (investigatordesigned questionnaire; $\alpha=0.81,{ }^{7}$ fertility-related distress (Reproductive Concerns after Cancer Scale (RCACS ${ }^{44}$ ) and general distress (ie, the Impact of Events ScaleRevised (IES-R).$^{45}$ Process variables will be evaluated to characterise change over time in relation to intervention outcomes and include perceptions of infertility/ infertility risk (eg, controllability; Illness Perceptions Questionnaire-Revised (IPQ-R), ${ }^{46}$ general self-efficacy (PROMIS Self-Efficacy General (PROMIS-G), ${ }^{47}$ selfefficacy for managing difficult emotions in the context of chronic health conditions/risks (PROMIS Self-Efficacy for Managing Emotions short form (PROMIS-SE) ${ }^{48}$ and self-efficacy for managing health problems attributed to website use $\left(\mathrm{eHIQ}^{49}\right)$. Data on sociodemographic and medical characteristics, preference for managing uncertainty in the context of cancer (Uncertainty Management Preference scale ${ }^{50}$ ), health literacy (young adult screener tool $^{51}$ and screener for detecting inadequate health literacy) ${ }^{52}$ cancer-related financial toxicity (Comprehensive Score for Financial Toxicity ${ }^{53}$ ) and general financial well-being (InCharge Financial Distress/Financial Wellbeing scale $)^{54}$ will be collected to describe the study sample and evaluate as relevant covariates. Investigator-designed questions will ask participants to report interventionprompted actions and concomitant care such as seeing a fertility specialist (reproductive endocrinologist) or accessing medical or supportive care resources suggested by the website. 


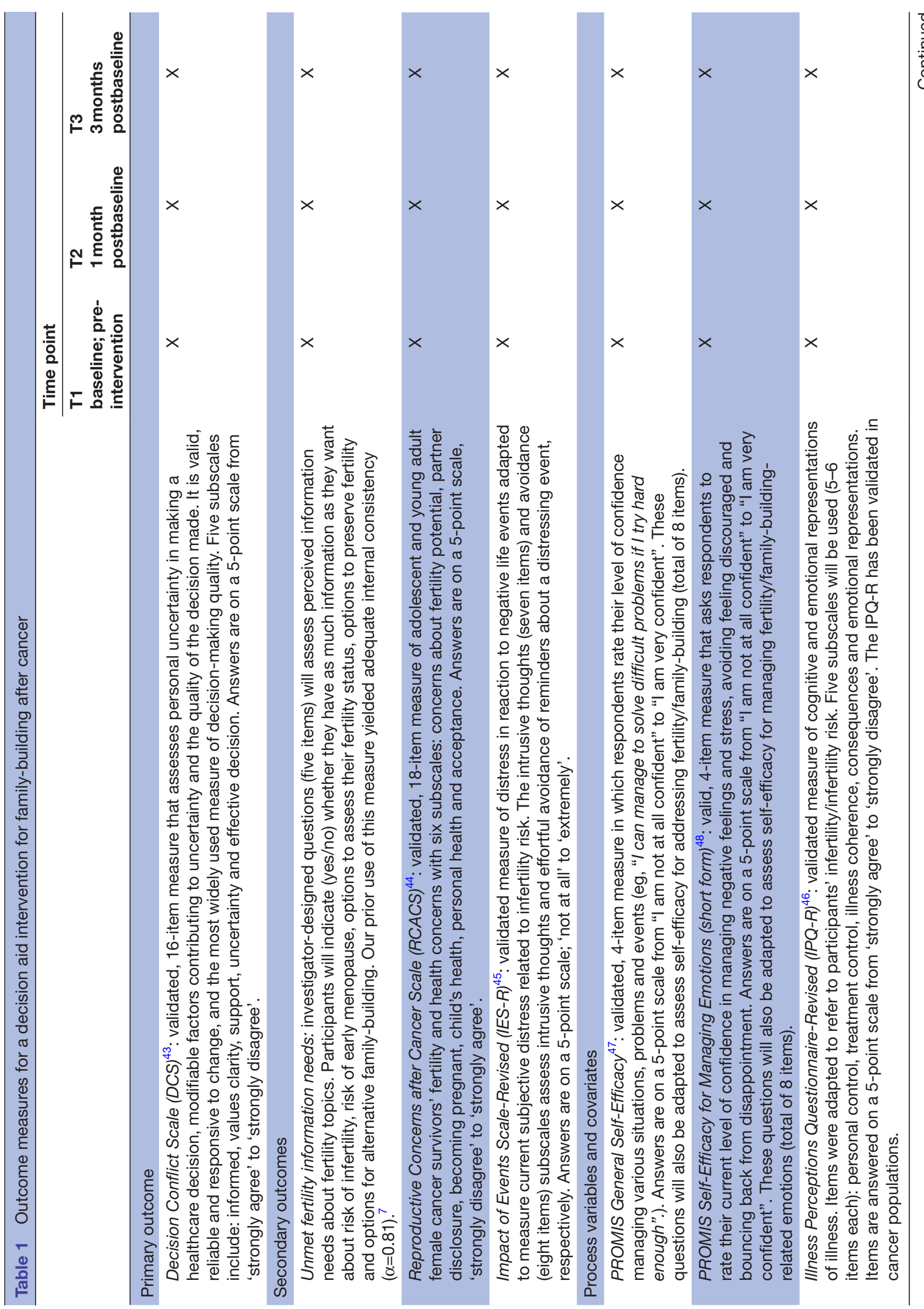




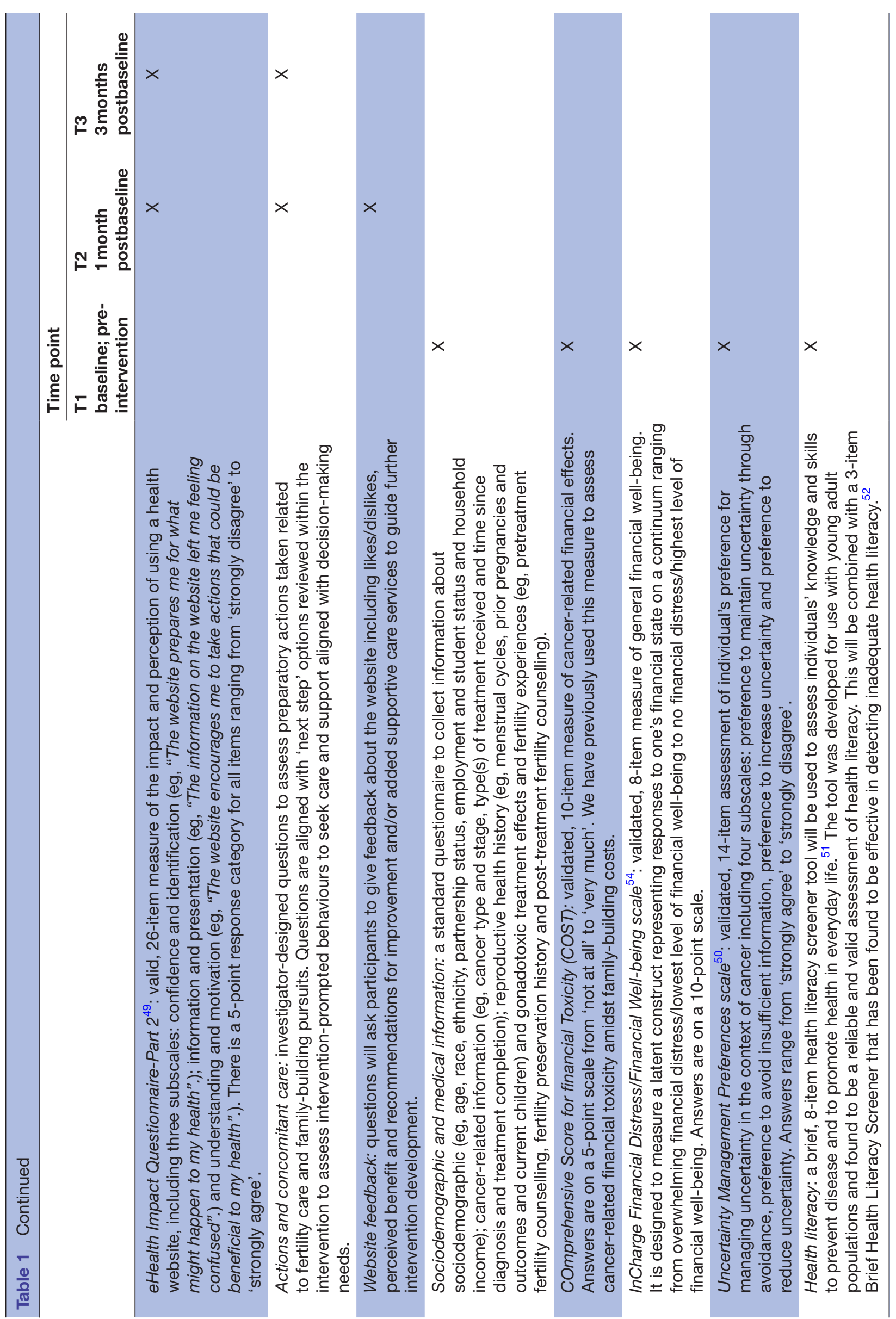




\section{Sample size calculation}

One of the main objectives of this pilot study is to obtain preliminary effect size estimates to inform a randomised clinical trial. With a projected $20 \%$ attrition rate, we expect $80 \%$ power to detect an effect size of 0.3 for the T1-T2 change in DCS, with a two-sided paired t-test with a 0.05 significance level. We based this on a decision aid intervention for fertility preservation, ${ }^{55}$ which reported $14 \%$ attrition and DCS means at baseline $(\mathrm{M}=51.6, \mathrm{SD}=32.8)$ and 1 month postintervention $(\mathrm{M}=16.6, \mathrm{SD}=26.5)$. We assumed the same DCS baseline mean, but were conservative and assumed higher attrition, higher DCS mean at follow-up (ie, smaller intervention effect; $\mathrm{M}=40, \mathrm{SD}=26.5$ ) and correlation coefficient between measurements to be 0.3 , so that the effect size will be 0.33 , representing a moderate effect size based on Cohen's taxonomy.

\section{Data analysis}

For aim 1, feasibility and acceptability metrics will be assessed using descriptive statistics of rates of eligibility, enrolment, attrition, reasons for refusal and completion rates of surveys. Independent means t-tests and $\chi^{2}$ tests will compare acceptors/decliners and completers/noncompleters. Web analytics (ie, Google analytics) will track web page access, time spent on modules, click stream data and visitor segmentation. Aggregated and real-time access to these data will help us determine use of the website so that we can make website design adjustments as needed. Access to the website and user engagement will be tracked for the entire study period. Survey data of selfreported website use will be compared with web analytics and used to further assess acceptability of the intervention and provide information on how participants engage with the intervention over time. Quantitative and qualitative feedback on the website (eg, usability, helpfulness) will be reviewed.

For aim 2, preliminary analyses will examine the distributional qualities for each outcome variable, extent of missing data and satisfaction of assumptions of randomness (ie, missing at random [MAR], missing completely at random $[\mathrm{MCAR}])$, if data imputation is warranted. Descriptive statistics will evaluate sample characteristics. To obtain an effect size estimate of intervention-related effects on decisional conflict, pairwise t-tests will evaluate change in DCS mean scores from T1 to T2. A two-sided test of the within-subject difference in adjusted means will test the null hypothesis $\left(H_{0}: \mu_{1}=\mu_{2}\right)$ at $\alpha=0.05$. Rejection of the null hypothesis will indicate a significant withinsubject DCS mean change from T1 to T2 $\left(\mathrm{H}_{1}: \mu_{1}>\mu_{2}\right)$. Effect size estimates (Cohen's d) will be calculated and reported.

On an exploratory level, a linear mixed-effects model will assess the trajectory of decisional conflict across all time points (T1-T3) and controlling for relevant covariates. Covariates will be evaluated to identify factors that relate to decisional conflict at baseline and intervention response. Post hoc tests will further characterise the trajectory of decisional conflict and compare T1-T2 and T2-T3 differences in mean change scores. Similar procedures will analyse group differences from (T1-T2) and trajectories of unmet information needs, fertility distress (RCACS), general distress (IES-R), illness perceptions (IPQ-R) and different aspects of self-efficacy (PROMIS-G, PROMIS-SE and $\mathrm{eHIQ}$ ) as secondary outcomes and process variables. Descriptive statistics will evaluate actions taken related to family-building decisional processes aligned with website suggestions and concomitant care.

\section{Ethics and dissemination}

We expect to conduct this study from January 2020 to July 2023. The study asks participants to review information about cancer-related infertility risk and family-building options. Based on participants' feedback obtained during the collection of our pilot data in three separate studies, we believe that many young women appreciate the opportunity to learn about their fertility, gonadotoxic treatment effects and options for family-building; and appreciate the opportunity to participate in research that aims to improve cancer and reproductive healthcare for others. Nevertheless, it is possible that participants may experience psychological distress reviewing information or answering questions about fertility and family-building while participating in study activities. There is also a minimal risk associated with data security such as loss of confidentiality of identifiable information. We have established planned procedures for protecting against and minimising all potential risks. Steps to mitigate risks will include:

1. All study materials will emphasise that eligibility information (ie, reason for contacting survivors to invite participation) was obtained based on population-level statistics (eg, treatment side effect risk profile) and does not reflect patients' actual fertility status or reproductive health. This will be reiterated during all conversations with participants including recruitment and consenting discussions.

2. The PI (Benedict), a licensed clinical psychologist with expertise in psycho-oncology, will be available for emergency consultation if needed.

3. Participants will be informed that they may skip questions they are uncomfortable answering or withdraw from the study at any time.

4. Participants will be informed that they may be withdrawn from the study for one or more of the following reasons: failure to follow instructions, indication that continued participation could be harmful, the study is cancelled, administrative reasons or unanticipated circumstances.

5. Referrals to specialised services within the Stanford Health system will be made to those who are local and interested. Possible referrals will include to the Stanford Fertility Center, the Stanford Adolescent and Young Adult Cancer programme or the Stanford Cancer Survivorship Programme. Referrals to online resources will be made to those not in geographic proximity. 
6. All study materials were reviewed by an interdisciplinary team of clinicians and researchers with expertise in young adult oncology and oncofertility.

7. All study data including protected health information (PHI) data will be stored on secure drives on institution servers, according to Stanford policies for the protection of human subjects and HIPPA-compliant security and data storage. Only IRB-approved study team members will have access to PHI data. PHI data collected during recruitment for potential participants that do not enrol in the study will be destroyed.

Only the study team (PI and co-investigators; IRBapproved) will have access to the final dataset. The PI will lead primary analyses to achieve study aims. These ethical safeguards are consistent with IRB-approved recommendations for minimal risk studies.

Results of this study will be published in peer-reviewed journals and presented at academic conferences. Publication/presentation of findings will include authors who contribute substantially to the study design, data collection, analysis and reporting. Study funders (National Cancer Institute) will not have authority over study activities including interpretation of data or the presentation/ publication of findings.

We will also make efforts to disseminate study findings through multichannel connection points with the young adult cancer community, including presenting at patient conferences, online patient events and through social media outlets. Given our ongoing collaborations with several young adult cancer organisations (eg, Stupid Cancer, The Samfund and Lacuna Loft), we will establish feasible and appropriate ways to increase awareness among young adult cancer survivors about this research and implications for fertility-related survivorship care.

There are no plans to grant public access to participantlevel data or statistical code.

\section{Patient and public involvement}

The work was supported by patient research partners who provided input to the programme of research. The intervention was developed based on patient-centred principles in which patients were included in all steps of the research and development processes. Patient research partners were consulted to refine the research question and confirm the overall objectives of the study. Patients also partnered with us for the design of the study, the informational material to support the intervention, and the burden of the intervention from the patient's perspective. The PI (Benedict) is a member of the Stanford Adolescent and Young Adult Cancer Patient and Family Advisory Committee in which study progress is discussed at monthly meetings and feedback from patient members is encouraged. At the end of the study, patient research partners will be invited to comment on the findings and contribute to the dissemination plan.

\section{DISCUSSION}

Young adult cancer survivors are an underserved patient group characterised by disparities in service delivery and outcomes. ${ }^{1}$ Our overarching goal is to develop an intervention to support survivors in achieving their familybuilding goals post-treatment, thereby filling a critical unmet service need in young adult survivorship care. This study will pilot test a decision aid intervention, grounded in theory and based on extensive pilot work, that is the first to comprehensively address this gap. Findings will provide important information for the development of a web-based decision support intervention. Ultimately, the intervention aims to support YA-F survivors in achieving their reproductive and parenthood goals after cancer by making informed decisions and through early consideration of preparatory actions to plan for medical, emotional, financial and legal challenges associated with ART, surrogacy and adoption.

\section{Strengths and limitations}

Addressing fertility is considered a priority by young adult patients with cancer and by clinical organisations. ${ }^{14}$ Research has focused on improving clinical services at the point of diagnosis and supporting patients through pretreatment fertility preservation. However, regardless of fertility preservation history, follow-up fertility services and support for family-building are also necessary. ${ }^{56}$ Arguably, such services are even more important in the post-treatment phase to address the numerous challenges inherent to ART, surrogacy and adoption, particularly as survivors report feeling largely uninformed about alternative family-building options aside from pregnancy. ${ }^{757}$ Future research based on these findings has great potential to improve cancer survivorship care and quality of life among young women who have completed gonadotoxic cancer treatment and hope to pursue motherhood.

Although there are significant strengths to this study, we also acknowledge limitations. As this is a pilot study, we will not be measuring long-term changes in outcome variables and thus, will not be able to detect if family-building decisions need to be revisited and renegotiated over time based on health outcomes or changing priorities. Conceptually, the decision aid tool may be used iteratively as new or evolving decision support needs arise, but we will be limited in our capacity to capture this. Likewise, it may be that decision conflict and distress increase initially after learning of the challenges associated with family-building options and it is unknown whether a longer timeline may be needed to capture intervention effects. Although we have conducted formative groundwork examining patient-reported decision support needs and preferences, findings may indicate that YA-Fs (or a subgroup) need more comprehensive support services. In this case, data will be used to develop supplemental components to the intervention using a stepped care model, such as offering personalised guidance or more extensive financial counselling. We are also unable to address system, 
provider and partner/family-level factors that may affect YA-Fs' decision-making. While we will focus on patient factors in this study and propose a patient-level intervention strategy, we will collect limited supplemental data on other levels, which may inform future research.

\section{Next steps}

When the pilot study is completed, the investigators will review the findings to determine feasibility, acceptability and effect size estimates of the decision aid intervention. Based on these results, the investigators plan to conduct a larger randomised controlled trial (RCT). This RCT will assess website use and outcomes over a longer time period to determine how survivors' needs may evolve over time, whether the website is suited to provide ongoing support and benefit and to explore whether there are latent subgroups that respond to the intervention differently or are in need of support. Findings may indicate additional intervention components are needed, such as parallel resources for providers, which may be considered for future testing. Future work will examine how the decision aid and planning tool may be adapted for other decision makers (eg, partners) and decision-making contexts such as in clinical settings to facilitate patient-provider communication and shared decision-making. In particular, a shared decision-making tool may be useful beyond oncologic settings as many post-treatment survivors do not regularly see their oncologist but may see other clinicians more regularly such as primary care physicians or gynaecologists. Findings may also inform decision support tools to be used by male survivors and/or among couples facing infertility/infertility risk and family-building challenges. As medical information and research findings are always evolving, the investigators intend to update the content and functionality of the website periodically to optimise its use and effectiveness.

\section{Author affiliations}

${ }^{1}$ Stanford University School of Medicine, Stanford, California, USA

${ }^{2}$ Stanford Cancer Institute, Palo Alto, California, USA

${ }^{3}$ Hunter College and The Graduate Center, City University of New York (CUNY), New York City, New York, USA

${ }^{4}$ Lucile Packard Children's Hospital Stanford, Palo Alto, California, USA

${ }^{5}$ Center for Health Innovation and Outcomes Research, Feinstein Institute for Medical Research, Northwell Health, Manhasset, New York, USA

Contributors CB contributed to the conceptualisation of the study objectives, study design and writing of the manuscript. JSF contributed to the conceptualisation of the study objectives, study design and editing of the manuscript. LS, PS and DS contributed to the study design and editing of the manuscript. MD contributed to the conceptualisation of the study objectives, study design and editing of the manuscript.

Funding This work was supported by a grant from the National Cancer Institute (K07CA229186, PI: Benedict).

Competing interests None declared.

Patient consent for publication Not required.

Ethics approval The study was approved by the Stanford Research Ethics Board (IRB\# 52143, V.1) and the Scientific Review Committee of the Stanford Cancer Institute.

Provenance and peer review Not commissioned; externally peer reviewed.
Open access This is an open access article distributed in accordance with the Creative Commons Attribution Non Commercial (CC BY-NC 4.0) license, which permits others to distribute, remix, adapt, build upon this work non-commercially, and license their derivative works on different terms, provided the original work is properly cited, appropriate credit is given, any changes made indicated, and the use is non-commercial. See: http://creativecommons.org/licenses/by-nc/4.0/.

ORCID iD

Catherine Benedict http://orcid.org/0000-0001-9622-249X

\section{REFERENCES}

1 Coccia PF, Pappo AS, Beaupin L, et al. Adolescent and young adult oncology, version 2.2018, NCCN clinical practice guidelines in oncology. J Nat/ Compr Canc Netw 2018;16:66-97.

2 Keegan THM, Ries LAG, Barr RD, et al. Comparison of cancer survival trends in the United States of adolescents and young adults with those in children and older adults. Cancer 2016;122:1009-16.

3 Lewin J, Ma JMZ, Mitchell L, et al. The positive effect of a dedicated adolescent and young adult fertility program on the rates of documentation of therapy-associated infertility risk and fertility preservation options. Support Care Cancer 2017;25:1915-22.

4 Irene Su H, Lee YT, Barr R. Oncofertility: meeting the fertility goals of adolescents and young adults with cancer. Cancer $J$ 2018;24:328-35.

5 Kim J, Mersereau JE, Su HI, et al. Young female cancer survivors' use of fertility care after completing cancer treatment. Support Care Cancer 2016;27:3191-9.

6 Geue K, Richter D, Schmidt R, et al. The desire for children and fertility issues among young German cancer survivors. J Adolesc Health 2014;54:527-35.

7 Benedict C, Thom B, N. Friedman D, et al. Young adult female cancer survivors' unmet information needs and reproductive concerns contribute to decisional conflict regarding posttreatment fertility preservation. Cancer 2016;122:2101-9.

8 Benedict C, Shuk E, Ford JS. Fertility issues in adolescent and young adult cancer survivors. J Adolesc Young Adult Oncol 2016;5:48-57.

9 Ehrbar V, Urech C, Tschudin S. Fertility decision-making in cancer patients - current status and future directions. Expert Rev Qual Life Cancer Care 2018;3:113-9.

10 Woodard TL, Hoffman AS, Covarrubias LA, et al. The pathways fertility preservation decision aid website for women with cancer: development and field testing. J Cancer Surviv 2018;12:101-14.

11 Stark SS, Natarajan L, Chingos D, et al. Design of a randomized controlled trial on the efficacy of a reproductive health survivorship care plan in young breast cancer survivors. Contemp Clin Trials 2019;77:27-36.

12 Bradford A, Woodard TL. Novel psychological intervention for decision support in women considering fertility preservation before cancer treatment. J Adolesc Young Adult Oncol 2017;6:348-52.

13 Wang Y, Anazodo A, Logan S. Systematic review of fertility preservation patient decision AIDS for cancer patients. Psychooncology 2019;28:459-67.

14 Logan S, Perz J, Ussher JM, et al. A systematic review of patient oncofertility support needs in reproductive cancer patients aged 14 to 45 years of age. Psychooncology 2018;27:401-9.

15 Ehrbar V, Urech C, Rochlitz C, et al. Randomized controlled trial on the effect of an online decision aid for young female cancer patients regarding fertility preservation. Hum Reprod 2019;34:1726-34.

16 Stacey D, Kryworuchko J, Belkora J, et al. Coaching and guidance with patient decision AIDS: a review of theoretical and empirical evidence. BMC Med Inform Decis Mak 2013;13:S11.

17 Raghunathan NJ, Benedict C, Thom B, et al. Young adult female cancer survivors' concerns about future children's health and genetic risk. J Adolesc Young Adult Oncol 2018;7:125-9.

18 Benedict C, Thom B, Friedman DN, et al. Fertility information needs and concerns post-treatment contribute to lowered quality of life among young adult female cancer survivors. Support Care Cancer 2018;26:2209-15.

19 Benedict C, McLeggon J-A, Thom B, et al. "Creating a family after battling cancer is exhausting and maddening": Exploring real-world experiences of young adult cancer survivors seeking financial assistance for family building after treatment. Psychooncology 2018;27:2829-39.

20 Benedict C, Thom B, Kelvin JF. Fertility preservation and cancer: challenges for adolescent and young adult patients. Curr Opin Support Palliat Care 2016;10:87-94.

21 Kelvin JF, Thom B, Benedict C, et al. Cancer and fertility program improves patient satisfaction with information received. $J$ Clin Oncol 2016;34:1780-6. 
22 Benedict C, Thom B, Kelvin JF. Young adult female cancer survivors' decision regret about fertility preservation. J Adolesc Young Adult Oncol 2015;4:213-8.

23 Thom B, Benedict C, Friedman DN, et al. The intersection of financial toxicity and family building in young adult cancer survivors. Cancer 2018;124:3284-9.

24 Hagger MS, Koch S, Chatzisarantis NLD, et al. The common sense model of self-regulation: meta-analysis and test of a process model. Psychol Bull 2017;143:1117-54.

25 Donovan EE, Brown LE, LeFebvre L, et al. "The uncertainty is what is driving me crazy": the tripartite model of uncertainty in the adolescent and young adult cancer context. Health Commun 2015;30:702-13.

26 Sepucha KR, Borkhoff CM, Lally J, et al. Establishing the effectiveness of patient decision AIDS: key constructs and measurement instruments. BMC Med Inform Decis Mak 2013;13:S12.

27 U.S. department of health and human services. HHS web standards and usability guidelines. HHS.gov. Available: https://www.hhs.gov/ [Accessed 24 May 2019].

28 Chan A-W, Tetzlaff JM, Altman DG, et al. SPIRIT 2013 statement: defining standard protocol items for clinical trials. Ann Intern Med 2013;158:200-7.

29 Warner EL, Kent EE, Trevino KM, et al. Social well-being among adolescents and young adults with cancer: a systematic review. Cancer 2016;122:1029-37.

30 Armuand GM, Wettergren L, Rodriguez-Wallberg KA, et al. Desire for children, difficulties achieving a pregnancy, and infertility distress 3 to 7 years after cancer diagnosis. Support Care Cancer 2014;22:2805-12.

31 Armuand GM, Wettergren L, Rodriguez-Wallberg KA, et al. Women more vulnerable than men when facing risk for treatment-induced infertility: a qualitative study of young adults newly diagnosed with cancer. Acta Oncol 2015;54:243-52.

32 Armuand GM, Rodriguez-Wallberg KA, Wettergren L, et al. Sex differences in fertility-related information received by young adult cancer survivors. JCO 2012;30:2147-53.

33 Pera A. Psychopathological processes involved in social comparison, depression, and Envy on Facebook. Front Psychol 2018;9:22.

34 Baker DA, Algorta GP. The relationship between online social networking and depression: a systematic review of quantitative studies. Cyberpsychol Behav Soc Netw 2016;19:638-48.

35 Durand M-A, Witt J, Joseph-Williams N, et al. Minimum standards for the certification of patient decision support interventions: feasibility and application. Patient Educ Couns 2015;98:462-8.

36 Coulter A, Stilwell D, Kryworuchko J, et al. A systematic development process for patient decision AIDS. BMC Med Inform Decis Mak 2013;13:S2.

37 Decision Aid Toolkit - Patient Decision Aids - Ottawa Hospital Research Institute. Available: https://decisionaid.ohri.ca/resources. html [Accessed 4 Jun 2019].

38 Sepucha KR, Abhyankar P, Hoffman AS, et al. Standards for universal reporting of patient decision aid evaluation studies: the development of SUNDAE checklist. BMJ Qual Saf 2018;27:380-8.

39 Hou S-I. Health literacy online: a guide to writing and designing easyto-use health web sites. Health Promot Pract 2012;13:577-80.
40 Turner-McGrievy GM, Hales SB, Schoffman DE, et al. Choosing between responsive-design websites versus mobile apps for your mobile behavioral intervention: presenting four case studies. Trans/ Behav Med 2017;7:224-32.

41 Docherty SL, Crane S, Haase JE, et al. Improving recruitment and retention of adolescents and young adults with cancer in randomized controlled clinical trials. Int J Adolesc Med Health 2019.

42 Topolovec-Vranic J, Natarajan K. The use of social media in recruitment for medical research studies: a scoping review. J Med Internet Res 2016;18:e286.

43 O'Connor AM. Validation of a decisional conflict scale. Med Decis Making 1995;15:25-30.

44 Gorman JR, Su HI, Pierce JP, et al. A multidimensional scale to measure the reproductive concerns of young adult female cancer survivors. J Cancer Surviv 2014;8:218-28.

45 Weiss DS. The Impact of Event Scale: Revised. In: Wilson JP, Tang CS, eds. Cross-Cultural Assessment of Psychological Trauma and PTSD. International and Cultural Psychology Series. Boston, MA: Springer US, 2007: 219-38.

46 Moss-Morris R, Weinman J, Petrie K, et al. The revised illness perception questionnaire (IPQ-R). Psychol Health 2002;17:1-16.

47 Salsman JM, Schalet BD, Merluzzi TV, et al. Calibration and initial validation of a general self-efficacy item bank and short form for the NIH PROMIS®. Qual Life Res 2019;28:2513-23.

48 Gruber-Baldini AL, Velozo C, Romero S, et al. Validation of

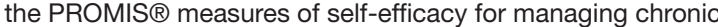
conditions. Qual Life Res 2017;26:1915-24.

49 Kelly L, Ziebland S, Jenkinson C. The e-health impact questionnaire: developing a tool to measure the effects of using health-related websites. Value in Health 2013;16.

50 Carcioppolo N, Yang F, Yang Q, Reducing YQ. Reducing, maintaining, or escalating uncertainty? the development and validation of four uncertainty preference scales related to cancer information seeking and avoidance. J Health Commun 2016;21:979-88.

51 Abel T, Hofmann K, Ackermann S, et al. Health literacy among young adults: a short survey tool for public health and health promotion research. Health Promot Int 2015;30:725-35.

52 Chew LD, Bradley KA, Boyko EJ. Brief questions to identify patients with inadequate health literacy. Fam Med 2004;36:588-94.

53 de Souza JA, Yap BJ, Hlubocky FJ, et al. The development of a financial toxicity patient-reported outcome in cancer: the cost measure. Cancer 2014;120:3245-53.

54 Prawitz A, Garman ET, Sorhaindo B, et al. Incharge financial Distress/ Financial well-being scale: development, administration, and score interpretation. Journal of Financial Counseling and Planning 2006;17.

55 Peate M, Meiser B, Cheah BC, et al. Making hard choices easier: a prospective, multicentre study to assess the efficacy of a fertilityrelated decision aid in young women with early-stage breast cancer. Br J Cancer 2012;106:1053-61.

56 Murphy D, Orgel E, Termuhlen A, et al. Why healthcare providers should focus on the fertility of AYA cancer survivors: it's not too late! Front Oncol 2013;3:248.

57 Gorman JR, Whitcomb BW, Standridge D, et al. Adoption consideration and concerns among young adult female cancer survivors. J Cancer Surviv 2017;11:149-57. 